\title{
The impact of blood glucose on community-acquired pneumonia: a retrospective cohort study
}

\author{
Andreas Vestergaard Jensen (10), ${ }^{1,2}$ Gertrud Baunbæk Egelund ${ }^{1,2}$, \\ Stine Bang Andersen ${ }^{1,2}$, Pelle Trier Petersen ${ }^{1,2}$, Thomas Benfield $\mathbb{1}^{2,3}$, \\ Daniel Faurholt-Jepsen ${ }^{4,5}$, Gernot Rohde ${ }^{6,7}$ and Pernille Ravn ${ }^{1,2}$
}

Affiliations: ${ }^{1}$ Dept of Pulmonary and Infectious Diseases, Nordsjællands Hospital, Hillerød, Denmark.
${ }^{2}$ University of Copenhagen, Faculty of Health and Medical Sciences, Copenhagen, Denmark. ${ }^{3}$ Dept of
Infectious Diseases, Hvidovre Hospital, Hvidrove, Denmark. ${ }^{4}$ Dept of Infectious Diseases, Rigshospitalet,
Copenhagen, Denmark. ${ }^{5}$ University of Copenhagen, Dept of Nutrition, Exercise and Sports, Copenhagen,
Denmark. ${ }^{6}$ Dept of Respiratory Medicine, Maastricht University Medical Center, Maastricht, The Netherlands.
${ }^{7}$ CAPNETZ-Stiftung, Hannover, Germany.

Correspondence: Andreas Vestergaard Jensen, Dept of Pulmonary and Infectious Diseases, Nordsjællands Hospital-Hillerød, Dyrehavevej 29, 3400 Hillerød, Denmark. E-mail: avestjahotmail.com

ABSTRACT Hyperglycaemia is common in patients with community-acquired pneumonia (CAP) and is a predictor of severe outcomes. Data are scarce regarding whether this association is affected by diabetes mellitus (DM) and also regarding its importance for severe outcomes in hospital. We determined the impact of blood glucose on severe outcomes of CAP in hospital.

We studied 1318 adult CAP patients hospitalised at three Danish hospitals. The association between blood glucose and DM status and severe clinical outcome (admission to an intensive care unit (ICU) and/ or in-hospital mortality) was assessed by logistic regression. Models were adjusted for CURB-65 score and comorbidities.

$12 \%$ of patients had DM. In patients without DM an increase in admission blood glucose was associated with risk for ICU admittance (OR 1.25, 95\% CI 1.13-1.39), but not significantly associated with inhospital mortality (OR 1.10, 95\% CI 0.99-1.23). In patients with DM an increase in admission blood glucose was not associated with ICU admittance (OR 1.05, 95\% CI 1.00-1.12) or in-hospital mortality (OR $1.05,95 \%$ CI $0.99-1.12$ ).

An increase in admission blood glucose (only in patients without DM) was associated with a higher risk for ICU admittance and a trend towards higher in-hospital mortality. DM was not associated with a more severe outcome of CAP.

@ERSpublications

An increase in blood glucose marks severity of community-acquired pneumonia in patients without diabetes mellitus http://ow.ly/3omE30c0whm

Cite this article as: Jensen AV, Egelund GB, Andersen SB, et al. The impact of blood glucose on community-acquired pneumonia: a retrospective cohort study. ERJ Open Res 2017; 3: 00114-2016 [https://doi.org/10.1183/23120541.00114-2016].

Received: Oct 112016 | Accepted after revision: April 212017

Support statement: The study has received support from The Christenson-Cesons Family Foundation, Fru Olga Bryde Nielsens Foundation and Kaptajnløjtnant Harald Jensens og Hustrus Foundation. The funding parties did not have any influence on the study design, execution of the study or interpretation of the results. Funding information for this article has been deposited with the Crossref Funder Registry.

Conflict of interest: Disclosures can be found alongside this article at openres.ersjournals.com

Copyright $\odot$ ERS 2017. This article is open access and distributed under the terms of the Creative Commons Attribution Non-Commercial Licence 4.0. 


\section{Introduction}

Hyperglycaemia is frequent at admission in patients with and without diabetes mellitus (DM) [1]. It may be secondary to DM (diagnosed or undiagnosed) or stress. Stress-induced hyperglycaemia is a result of an increase in catecholamines, glucagon, growth hormone, proinflammatory cytokines and increased peripheral insulin resistance in response to physiological stress [2]. Systemic bacterial and viral infections are potent triggers of stress hyperglycaemia and this may also be the case for pneumonia.

Community-acquired pneumonia (CAP) remains a leading cause of hospitalisation and mortality worldwide [3, 4]. The incidence of hospitalisations caused by pneumonia has increased steadily worldwide [5]. Rates in Denmark have increased from 288 to 809 per 100000 person-years over recent decades [6]. Previous studies have suggested that hyperglycaemia may be associated with severe outcome in a range of conditions, including sepsis $[1,7,8]$. In some CAP studies, an association with higher mortality has also been reported [9-12], while others have shown no association [13, 14]. Most studies have focused on the association between hyperglycaemia and long-term mortality (28-90 day mortality) after an episode of CAP $[10,13]$. Thus, there is a need for further analysis on the impact of hyperglycaemia on morbidity and mortality associated with CAP, and particularly in-hospital mortality.

The main objective of the present study was to determine the impact of blood glucose levels on severe outcomes of CAP, defined as admission to an intensive care unit (ICU) and in-hospital mortality. Secondarily, we aimed to determine the impact of blood glucose levels on the clinical course of CAP.

\section{Methods}

\section{Study population}

We retrospectively included adult patients admitted to three Danish hospitals (one teaching and two smaller referral hospitals) in the CAP-NORTH cohort. This cohort has been described in detail elsewhere [15]. In brief, data were collected between January 1, 2011 and June 30, 2012. The hospitals served a population of approximately 360000 citizens. The hospitals received all patients in need of acute hospital admittance because of the free access to treatment in public hospitals and absence of private medical emergency hospitals. Thus the study covered all patients residing in the catchment area of these hospitals. Patients admitted with pneumonia were identified based on the International Classification of Diseases 10th revision (ICD-10) codes noted at discharge. All patients who had one of the following ICD-10 codes either as primary or secondary diagnosis were evaluated for inclusion: pneumonia J10.0, J11.0 and J12.X-J18.x, J69.X, Mycoplasma pneumoniae B96.0, Klebsiella pneumoniae B96.1, Ornithosis A70.x and Legionellosis A481.

Patient files were reviewed to assess whether the study inclusion and exclusion criteria were fulfilled (table 1). In brief, CAP was considered in patients with symptoms of lower respiratory tract infection, a new infiltrate on chest radiography and no hospital admission within the last 28 days. Each patient could only be included once into the study and was registered at their first presentation with CAP [15]. Information on DM was retrieved from the patient files and the patients were categorised as DM if 1) DM was self-reported, 2) the attending physician stated that the patient had DM or 3) the patient received either oral or injectable anti-diabetes medication before admission.

\section{Data collection}

Baseline characteristics, comorbidity and clinical findings at admission were collected from patient files. Data were entered into the CAPNETZ database (www.capnetz.de) as well as into EpiData Entry 3.1 (www. epidata.dk). Data related to hospitalisation were collected, including the use of antibiotics, microbiological findings and length of hospital stay (LOS). Duration of antibiotic therapy included hospitalisation and discharge.

\section{TABLE 1 Inclusion and exclusion criteria}

\section{Inclusion criteria}

Age $\geqslant 18$ years

New infiltrate on chest radiography (confirmed by radiologist)

At least one of the following symptoms of lower respiratory tract infection

Cough

Purulent expectoration

Fever $\geqslant 38.3^{\circ} \mathrm{C}$ (rectal) or $\geqslant 37.8^{\circ} \mathrm{C}$ (ear)

Pathological lung auscultation
Exclusion criteria

Hospital admission within the last 28 days prior to admission due to pneumonia Active tuberculosis Missing information on prior medical history Immunosuppression, defined as

Treatment with corticosteroids ( $\geqslant 20 \mathrm{mg}$ prednisolone-equivalent/day $>14$ days) HIV infection

Received cancer chemotherapy within the last 28 days and had neutropenia (neutrophil granulocytes $<1000 \mu \mathrm{L}^{-1}$ )

Immunosuppression after organ or bone marrow transplantation 
Treatment length was registered as total length of antibiotic treatment (LOAB) and length of intravenous antibiotic treatment (LOIVAB). LOAB, LOIVAB and LOS were used as markers of the clinical course of CAP. As markers of severe outcome of CAP we considered admittance to intensive care unit (ICU) and/or death in hospital. Admission glucose was defined as the first measured glucose value on the admission day. We used CURB-65 as a severity score [16].

Except for comorbidities, data not mentioned/recorded in patients' files were noted as missing.

\section{Ethics}

The study was approved by the Danish Health and Medicines Authority (3-3013-340/1/) and the Danish Data Protection Agency (HIH-2013-017). Informed consent is not required under Danish legislation for register-based studies.

\section{Statistics}

Data were analysed using SAS Enterprise Guide version 5.1 (SAS Institute A/S). Data were described as counts (\%) for categorical variables and either means (standard deviation (SD)) or medians (interquartile range (IQR)) for continuous variables as appropriate. The t-test, Wilcoxon rank sum test, $\chi^{2}$ and Fisher's exact test were used to test for differences between participants with and without DM, as appropriate. Where relevant, we adjusted for multiple comparisons using the Bonferroni correction. Patients who died in hospital were reported as having missing LOS.

Due to the skewed distribution, the clinical course outcome variables (LOIVAB, LOS and LOAB) were log-transformed $(\ln )$ and the proposed predictors, blood glucose levels and DM (DM versus non-DM)

TABLE 2 Characteristics of patients with and without diabetes mellitus at time of admission

\begin{tabular}{|c|c|c|c|}
\hline & Diabetes & Nondiabetes & p-value \\
\hline Patients & $12.3(162)$ & $87.7(1156)$ & \\
\hline \multicolumn{4}{|l|}{ Demographic data } \\
\hline Age years & $73(65-80)$ & $71(58-82)$ & 0.06 \\
\hline Male & $64.2(104)$ & $45.1(521)$ & 0.001 \\
\hline Current smoker & $23.8(35)$ & 27.9 (295) & 0.30 \\
\hline Nursing home resident & $8.6(14)$ & $11.4(131)$ & 0.30 \\
\hline \multicolumn{4}{|l|}{ Comorbidities } \\
\hline Obstructive pulmonary disease & $23.6(38)$ & $17.9(205)$ & 0.08 \\
\hline Other pulmonary disease & $11.8(19)$ & 11.7 [135] & 0.98 \\
\hline Heart failure & $16.7(27)$ & $7.4(86)$ & $<0.001$ \\
\hline Other cardiac disease & $27.2(44)$ & $14.1(163)$ & $<0.001$ \\
\hline Liver disease & $0.62(1)$ & $0.95(11)$ & 1.0 \\
\hline Kidney disease & $8.6(14)$ & 2.3 (27) & $<0.001$ \\
\hline Cerebrovascular disease & $13.0(21)$ & $9.9(114)$ & 0.22 \\
\hline Other neurological disease & 1.9 (3) & $4.3(50)$ & 0.13 \\
\hline Malignancy & $7.4(12)$ & $9.3(107)$ & 0.44 \\
\hline Admission blood glucose ${ }^{\#} \mathrm{mM}$ & $9.2(7.3-13.5)$ & $6.8(6.0-7.9)$ & $<0.001$ \\
\hline Blood glucose $\leqslant 6 \mathrm{mM}$ & $8.8(14)$ & $28.0(317)$ & $<0.001^{+}$ \\
\hline Blood glucose $6.1-11 \mathrm{mM}$ & $52.8(84)$ & $68.8(780)$ & $<0.001^{+}$ \\
\hline Blood glucose $\geqslant 11.1 \mathrm{mM}$ & $38.4(61)$ & $3.2(36)$ & $<0.001^{+}$ \\
\hline \multicolumn{4}{|l|}{ CURB-65 } \\
\hline $0-1$ & $36.7(51)$ & 55.5 (553) & $<0.001^{+}$ \\
\hline 2 & 42.5 (59) & $27.2(271)$ & $<0.001^{+}$ \\
\hline $3-5$ & 20.9 (29) & $17.4(173)$ & $0.93^{+}$ \\
\hline \multicolumn{4}{|l|}{ CURB-65 parameters } \\
\hline Confusion & $13.1(21)$ & $14.4(162)$ & 0.68 \\
\hline Urea $>7.0 \mathrm{mM}$ & $57.2(91)$ & $37.2(424)$ & $<0.001$ \\
\hline Respiratory rate $\geqslant 30 \mathrm{~min}^{-1}$ & $16.3(22)$ & $10.9(112)$ & 0.11 \\
\hline Blood pressure & $15.7(25)$ & $15.3(172)$ & 0.89 \\
\hline Age $\geqslant 65$ years & $77.2(125)$ & $56.2(741)$ & 0.001 \\
\hline
\end{tabular}

Data are presented as $\%(n)$ or median (interquartile range). All variables had $<3 \%$ missing data except for current smokers $(n=113,8.5 \%)$ and CURB-65 $(n=182,13.8 \%)$. * : range 1.3-73.1 mM in diabetes patients and 3.2-21.2 $\mathrm{mM}$ in nondiabetes patients; " : systolic $<90$ and/or diastolic $\leqslant 60 \mathrm{mmHg}{ }^{+}$: Bonferroni adjusted. 
were assessed using a linear regression model. The association was assessed in univariate analyses and in a multivariate model to adjust for possible confounders. The regression coefficients were back-transformed $\left(100 \% \times\left(e^{\text {coefficient }}-1\right), 95 \%\right.$ CI $\left.100 \% \times\left(e^{\text {upper limit }}-1\right)-100 \% \times\left(e^{\text {lower limit }}-1\right)\right)$ to provide percentage effects.

We used logistic regression analyses to assess the risk of severe outcome, which was defined as ICU admittance, in-hospital mortality or combined risk (ICU admittance and/or in-hospital mortality). Admittance blood glucose (continuous variable) was used to predict severe outcome and, because the impact of blood glucose levels may be affected by diabetes status, we tested for an interaction between blood glucose level and diabetes status. To adjust for confounding, relevant predictors (CURB-65, chronic obstructive pulmonary disease (COPD), heart diseases and chronic kidney disease) were adjusted for. Additionally, we assessed the association between pre-admission diabetes status (categorical variable) on the risk of a severe outcome in a similar model, unadjusted and adjusted. All statistical comparisons were two-sided and carried out at the 0.05 significance level.

\section{Results}

The CAP-NORTH cohort comprised 1320 patients with CAP [15], and data for 1318 patients were available for this study. Two patients were not included due to missing information on prior medical history. In total, $12.3 \%$ (162/1318) of patients were known to have DM at admittance (table 2). There were no differences between patients with and without DM with respect to age, nursing home residency and smoking habits, but more patients with DM were male $(64.2 \%$ versus $45.1 \%, \mathrm{p}=0.001)$ and more often had chronic cardiac and kidney disease. Patients with DM were less likely to have received antibiotic treatment prior to admission $(29.4 \%$ versus $37.6 \%, \mathrm{p}=0.04)$. Patients with $\mathrm{DM}$ had higher median admittance blood glucose levels $\left(9.2 \mathrm{mmol} \cdot \mathrm{L}^{-1}\right.$ versus $\left.6.8 \mathrm{mmol} \cdot \mathrm{L}^{-1}(\mathrm{mM}), \mathrm{p}<0.001\right) .38 \%$ and $3 \%(\mathrm{p}<0.001)$ of patients with and without DM had admittance blood glucose $\geqslant 11.1 \mathrm{mM}$, respectively. At the time of admission, more patients with DM $(63.4 \%$ versus $44.6 \%, \mathrm{p}<0.001)$ had signs of moderate to severe pneumonia according to CURB-65 (table 2), predominantly due to age $>65$ years and urea $>7.0 \mathrm{mM}$.

\section{Association between admission blood glucose, DM and clinical course of CAP}

We assessed whether the clinical course of CAP was different in patients with DM than in patients without DM, and found that LOS and LOIVAB for patients with DM were longer (6 days versus 5 days, $\mathrm{p}<0.001 ; 4$ days versus 3 days, $\mathrm{p}=0.01$ ) (table 3 ), whereas LOAB was similar in patients with and without $\mathrm{DM}(\mathrm{p}=0.87)$. In order to adjust for potential confounders (CURB-65 score and comorbidities), we performed a linear regression analysis on log-transformed data, and found that LOS remained associated with DM, corresponding to a $22 \%$ (95\% CI 5.15-41.37\%) longer admission time compared to patients without DM. The association between DM and LOIVAB disappeared after adjusting (9\%, 95\% CI $-5.27-26.20 \%)$.

Subsequently, we assessed the influence of admission blood glucose in an adjusted linear regression model and found that for each $1 \mathrm{mM}$ increase in blood glucose, LOS increased by $2.8 \%$ (95\% CI $0.70-4.25 \%$ ) and the length of LOIVAB was extended by $1.4 \%$ (95\% CI 0.03-3.01\%). Blood glucose levels did not influence the LOAB.

\begin{tabular}{|c|c|c|c|}
\hline & Diabetes patients & Nondiabetes patients & p-value ${ }^{\#}$ \\
\hline Patients & $12.3(162)$ & $87.7(1156)$ & \\
\hline \multicolumn{4}{|c|}{ Clinical course days median (IQR) } \\
\hline LOSף & $6(4-10)$ & $5(3-8)$ & $<0.001$ \\
\hline LOAB & $10(8-12)$ & $10(8-12)$ & 0.87 \\
\hline LOIVAB & $4(2-7)$ & $3(2-6)$ & 0.01 \\
\hline \multicolumn{4}{|l|}{ Severe outcome } \\
\hline ICU admittance & $13.0(21)$ & $9.2(106)$ & 0.13 \\
\hline In-hospital mortality & $10.5(17)$ & $8.1(94)$ & 0.31 \\
\hline Any severe event ${ }^{+}$ & $19.8(32)$ & $13.9(161)$ & 0.05 \\
\hline \multicolumn{4}{|c|}{ 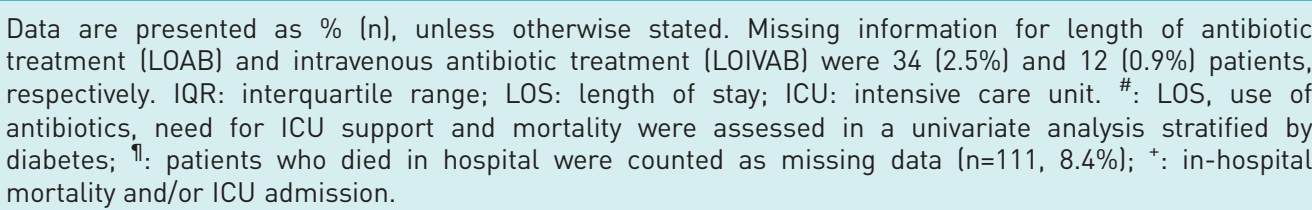 } \\
\hline
\end{tabular}




\section{Association between admission blood glucose levels and DM status and the risk of severe} outcome

In total, $19.8 \%(32 / 162)$ patients with and $13.9 \%(161 / 1156)$ without DM had a severe outcome during hospital admission. This included admission to an ICU (13.0\% versus $9.2 \%)$ and in-hospital death (10.5\% versus $8.1 \%$ ) (table 3 ).

The difference between patients with and without DM with regard to overall risk of severe outcome (ICU and/or in-hospital death) was borderline significant (OR 1.52, 95\% CI 0.998-2.32, p=0.051) by univariate analysis, whereas the differences in risk of either ICU admittance or death were not significant (table 4)

By multivariate analysis adjusting for CURB-65 and comorbidity (table 4), DM was no longer associated with any overall severe outcome (OR 1.43, 95\% CI 0.88-2.31), in-hospital death (OR 0.92, 95\% CI $0.47-1.79$ ) or ICU admittance (OR 1.62, 95\% CI 0.93-2.82).

We assessed the effect of blood glucose levels on severe outcome and found, in an unadjusted analysis, that the level of admittance blood glucose was associated with any severe outcome in both patients with and without DM. Specifically, for each $1 \mathrm{mM}$ increase in glucose level, the OR for ICU admittance was 1.26 (95\% CI 1.16-1.38) for patients without DM and 1.08 (95\% CI 1.02-1.14) for patients with DM (table 5). Adjustment for CURB-65 and comorbidity lowered the OR for patients with DM (OR 1.05, 95\% CI 1.00-1.12), but not for patients without DM (OR 1.25, 95\% CI 1.13-1.39).

Similarly, elevated admission blood glucose was associated with increased in-hospital mortality (OR 1.24, 95\% 1.13-1.35) in patients without DM, although this was partially confounded by CURB-65 and comorbidities (adjusted OR 1.10, 95\% CI 0.99-1.23). Known diabetes did not seem to be associated with excess risk of mortality.

There was a strong interaction between DM status and blood glucose levels (test for interaction, $\mathrm{p}=0.01$ ). This indicated that the association between blood glucose levels and ICU admittance depends on pre-admission DM status, and was more pronounced in patients without DM.

\section{Discussion}

We assessed the impact of blood glucose levels on severity of CAP. An increase in admission blood glucose was associated with a more severe outcome of CAP, predominantly in patients without DM. DM was not associated with in-hospital mortality or ICU admittance.

An increase in blood glucose at hospital admission was associated with prolonged LOS, LOIVAB and severe outcome (ICU and in-hospital mortality). Interestingly, the association between increasing blood glucose and LOS, LOIVAB and severe outcome was most pronounced for patients without DM. Diabetes was associated with prolonged LOS and LOIVAB, but only LOS remained significant after adjusting for confounders. Hyperglycaemia has been associated with excess mortality in patients admitted to ICUs [17] and in CAP patients with hyperglycaemia [9, 10, 18], although the literature is inconsistent [13, 14]. In 2012, LEPPER et al. [10] found, in a large prospective multicentre cohort study of CAP, that hyperglycaemia is strongly associated with 90 day mortality in patients without DM. The study by LEPPER et al. was a prospective study, where the patients were younger and one-third were not hospitalised. Despite differences in design and population, our study confirms this and other previous studies in showing that elevated blood glucose in patients without DM may be associated with severe outcome and mortality $[9,10,18]$.

\section{TABLE 4 Risk of severe outcome depending on diabetes status}

\begin{tabular}{|c|c|c|c|c|}
\hline & \multicolumn{2}{|c|}{ Crude analyses } & \multicolumn{2}{|c|}{ Multivariate analyses" } \\
\hline & OR $(95 \% \mathrm{CI})$ & p-value & Adjusted OR (95\% CI) & p-value \\
\hline \multicolumn{5}{|l|}{ Any severe outcome ${ }^{\pi}$} \\
\hline Nondiabetes patients & Ref. & & Ref. & \\
\hline Diabetes patients & $1.52(0.998-2.32)$ & 0.051 & $1.43(0.88-2.32)$ & 0.15 \\
\hline \multicolumn{5}{|l|}{ ICU admission } \\
\hline Nondiabetes patients & Ref. & & Ref. & \\
\hline Diabetes patients & $1.48(0.9-2.43)$ & 0.13 & $1.62(0.93-2.82)$ & 0.09 \\
\hline \multicolumn{5}{|l|}{ In-hospital mortality } \\
\hline Nondiabetes patients & Ref. & & Ref. & \\
\hline Diabetes patients & $1.33(0.77-2.29)$ & 0.31 & $0.92(0.47-1.79)$ & 0.80 \\
\hline
\end{tabular}




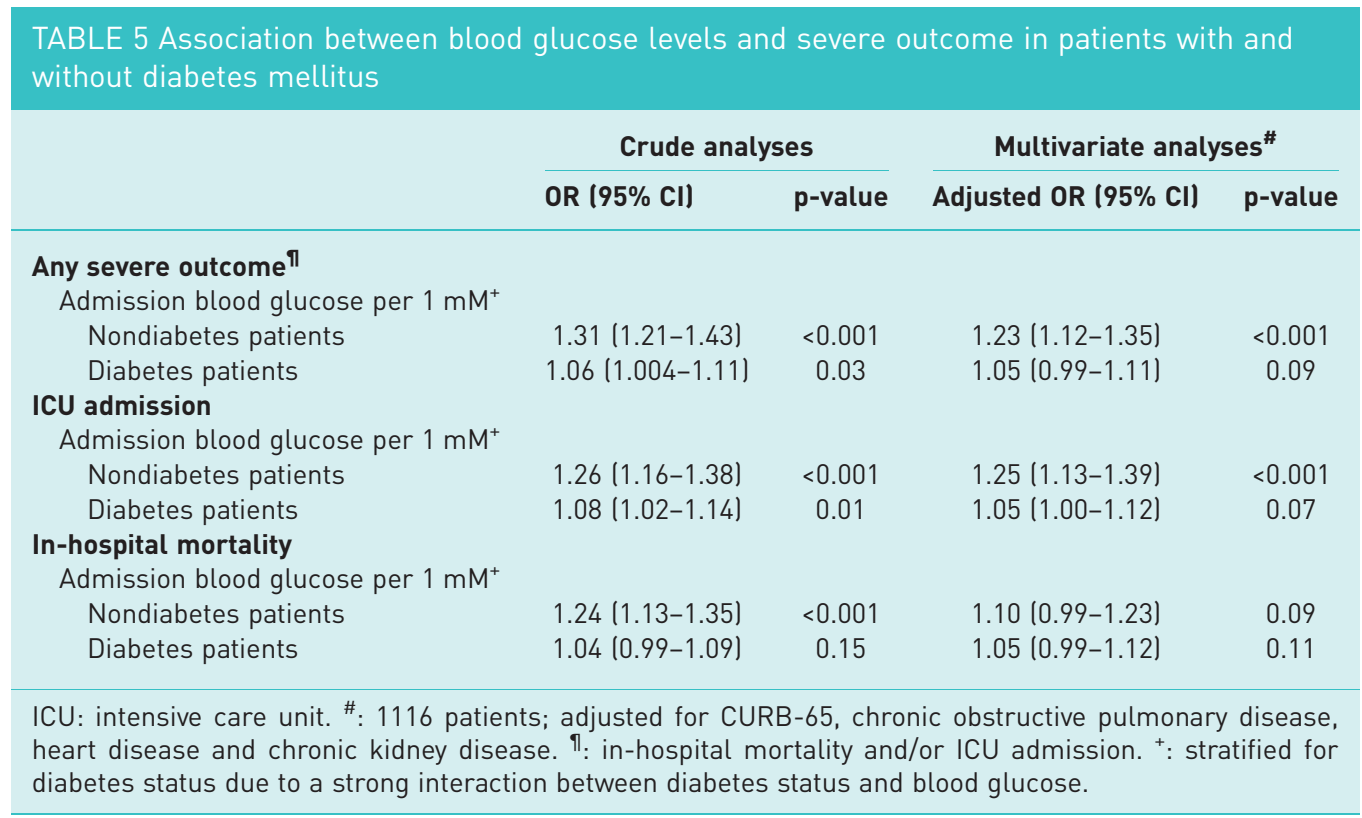

The association between an increase in admission blood glucose and a severe clinical course was most prominent in patients without DM. This is a somewhat counterintuitive finding, although the interaction between blood glucose and DM has been reported previously [10, 12, 19]. Explanations for the fact that hyperglycaemia in patients with DM apparently seemed less detrimental could be that patients with DM were more likely to have chronically elevated blood glucose levels than patients without DM. The stress hyperglycaemia response in patients with DM may therefore be overestimated due to higher glycaemic baseline levels compared to patients without DM. Moreover, clinicians may have had more focus on subsequent blood glucose regulation in patients with DM. However, it remains controversial whether tight glucose regulation is beneficial in the acute stages of diseases [20, 21].

In this cohort of hospitalised CAP patients, $12.3 \%$ had DM, which was comparable to recent studies of CAP, where the DM prevalence ranged from $9.8 \%$ to $21.4 \%[9,10,22,23]$. We did not find any association between DM and an increased risk of ICU admittance or in-hospital mortality, in spite of more severe disease at admittance based on the CURB-65 score. This is in contrast to previous studies reporting a higher mortality of patients with CAP and DM $[9,10,24,25]$. We found comparable in-hospital mortality for patients with and without DM (10.5\% and $8.1 \%$, respectively) whereas LEPPER et al. [10] reported an increased 90 day mortality (14.5\% versus 6\%) while FALGUERA et al. [25] reported an increased 30 day mortality (17\% versus $8 \%$ ) of patients with DM compared to patients without DM. Mortality was similar in patients with and without DM at days 30 and 90 (11\% and 15\%, respectively) in our cohort [15].

The comparable mortality of patients with and without DM may be due to several factors. Firstly, all hospitalised patients were included, regardless of severity or mental status, including patients admitted to the ICU and those who died within the initial $24 \mathrm{~h}$. These patients may be problematic to include in prospective studies due to difficulties in obtaining informed consent and a potential source of selection bias. Thus, patients included in our study were older and most likely more severely ill at admission, and the impact of DM was of minor importance compared to the overall severity.

Secondly, better overall management and earlier detection of patients with DM may result in lower than expected mortality from pneumonia. The age-standardised mortality rate among patients with DM in Denmark has been reduced by 22\%, from 1.90 in 1997 to 1.49 in 2012 [26]. In this regard it would have been interesting to determine if differences in glycated haemoglobin $\left(\mathrm{HbA}_{1 \mathrm{c}}\right)$ between the studies could confirm the hypothesis that better regulated DM patients had lower CAP-related mortality. These facts suggest that the excess mortality of pneumonia previously reported in diabetics is declining, while the average mortality in pneumonia is constant [6]. This may explain why we were not able to detect a difference in mortality between patients with and without DM.

Stress-induced hyperglycaemia is caused by a complex interplay of hormones such as catecholamines, growth hormone, cortisol and cytokines, which leads to excessive hepatic glucose production and insulin 
resistance [1]. Stress hyperglycaemia is mediated by greater inflammatory and neuroendocrine alterations than are expected in chronic hyperglycaemia, and some of these alterations might be subject for an intervention [1]. Microvascular endothelial dysfunction, e.g. as seen in multiorgan failure, has been associated with stress hyperglycaemia, and one study suggests that improved outcomes associated with intensive insulin therapy may in part be to endothelial protection $[1,27]$. Our study does not allow any conclusions on the beneficial effects of glycaemic control during severe infections. Therefore, studies in a non-ICU setting are required to determine whether optimal regulation of blood glucose levels improves (at least in the short term) outcomes in CAP.

\section{Limitations}

Firstly, our study was retrospective and observational, and we were not able to determine any causality between hyperglycaemia and severe outcome, and we cannot exclude that there was residual confounding that was not accounted for. Secondly, relying on self-reported DM gives rise to the problem that a proportion of our patients might have had unrecognised DM. Determination of HbA1c levels on admission could have solved this problem, but this information was not available. Thirdly, we cannot exclude that our sample was too small and hence lacked the power to detect a difference in mortality between patients with and without DM. Fourthly, glucocorticoids may increase blood glucose levels [28]. Therapy with glucocorticoids of more than $20 \mathrm{mg}$ daily for more than 14 days prior to admission was an exclusion criterion. Glucocorticoids are not recommended for CAP in Denmark. Only patients with suspicion of COPD exacerbations would have been offered prednisolone treatment for 10 days. We did not register the use of glucocorticoids during admission, but we did adjust our analysis for COPD. Fifthly, we did not register the time or nature of fluid resuscitation, which could have had an impact on blood glucose levels, and we did not have information on time from admission to antibiotic treatment. Sixthly, although our study in general had few missing values, nearly $14 \%$ of the patients were missing a CURB-65 score. Finally, some patients with CAP might have been missed if they were discharged with a diagnosis other than pneumonia.

\section{Strengths}

Due to the multicentre design, data were available for the entire geographic region and we believe that the cohort was representative of patients hospitalised with CAP in economically developed countries. Secondly, we evaluated both the primary and secondary diagnosis of pneumonia. This ensured that no patients were excluded due to severe illness or atypical presentation of CAP. Thirdly, by applying our inclusion and exclusion criteria we eliminated the potential confounding of hospital-acquired pneumonia and excluded non-pneumonia patients. Finally, the study reflected "real life", with no excessive focus on study patients or adherence to specific treatment regimens. This may allow for careful generalisation of the results.

\section{Conclusions}

An increase in admission blood glucose was an important clinical indicator of CAP severity, preferentially among patients without DM, while DM did not affect the severity of CAP in terms of risk of ICU admittance or in-hospital mortality. In patients without DM, an increase in blood glucose was associated with a higher risk for ICU admittance and a tendency towards an association with in-hospital mortality in line with recent evidence.

\section{Acknowledgements}

The authors acknowledge collaborators at CAPNETZ STIFTUNG, the CAPNETZ study group and the Pneumonia Research Group-Nordsjællands Hospital, including research nurse Gudrun Kaldan and medical student Sara Falk Jensen. The authors thank Lise Tarnow (Nordsjællands Hospital) for commenting on the manuscript.

\section{References}

Dungan KM, Braithwaite SS, Preiser J-C. Stress hyperglycaemia. Lancet 2009; 373: 1798-1807.

Marik PE, Bellomo R. Stress hyperglycemia: an essential survival response! Crit Care 2013; 17: 305.

Lozano R, Naghavi M, Foreman K, et al. Global and regional mortality from 235 causes of death for 20 age groups in 1990 and 2010: a systematic analysis for the Global Burden of Disease Study 2010. Lancet 2012; 380: 2095-2128.

4 Wroe PC, Finkelstein JA, Ray GT, et al. Aging population and future burden of pneumococcal pneumonia in the United States. J Infect Dis 2012; 205: 1589-1592.

5 Chalmers JD, Pletz MW, Aliberti S, eds. Community-Acquired Pneumonia (ERS Monograph). Sheffield, European Respiratory Society, 2014.

6 Søgaard M, Nielsen RB, Schønheyder HC, et al. Nationwide trends in pneumonia hospitalization rates and mortality, Denmark 1997-2011. Respir Med 2014; 108: 1214-1222.

7 Bruno A, Levine SR, Frankel MR, et al. Admission glucose level and clinical outcomes in the NINDS rt-PA Stroke Trial. Neurology 2002; 59: 669-674. 
8 Capes SE, Hunt D, Malmberg K, et al. Stress hyperglycaemia and increased risk of death after myocardial infarction in patients with and without diabetes: a systematic overview. Lancet 2000; 355: 773-778.

9 Kornum JB, Thomsen RW, Riis A, et al. Type 2 diabetes and pneumonia outcomes: a population-based cohort study. Diabetes Care 2007; 30: 2251-2257.

10 Lepper PM, Ott S, Nuesch E, et al. Serum glucose levels for predicting death in patients admitted to hospital for community acquired pneumonia: prospective cohort study. BMJ 2012; 344: e3397.

11 McAlister FA, Majumdar SR, Blitz S, et al. The relation between hyperglycemia and outcomes in 2,471 patients admitted to the hospital with community-acquired pneumonia. Diabetes Care 2005; 28: 810-815.

12 Rueda AM, Ormond M, Gore M, et al. Hyperglycemia in diabetics and non-diabetics: effect on the risk for and severity of pneumococcal pneumonia. J Infect 2010; 60: 99-105.

13 Eurich DT, Gamble JM, Marrie TJ, et al. Dysglycaemia and 90 day and 1 year risks of death or readmission in patients hospitalised for community-acquired pneumonia. Diabetologia 2010; 53: 497-503.

14 Bhattacharya RK, Mahnken JD, Rigler SK. Impact of admission blood glucose level on outcomes in community-acquired pneumonia in older adults. Int J Gen Med 2013; 6: 341-344.

15 Baunbæk Egelund G, Jensen AV, Andersen SB, et al. Penicillin treatment for patients with community-acquired pneumonia in Denmark: a retrospective cohort study. BMC Pulmon Med 2017; 17: 66.

16 Lim WS, van der Eerden MM, Laing R, et al. Defining community acquired pneumonia severity on presentation to hospital: an international derivation and validation study. Thorax 2003; 58: 377-382.

17 Plummer MP, Bellomo R, Cousins CE, et al. Dysglycaemia in the critically ill and the interaction of chronic and acute glycaemia with mortality. Intensive Care Med 2014; 40: 973-980.

18 Foltran F, Gregori D, Caropreso A, et al. Is blood glucose on admission a predictor of mortality in adult acute pneumonia? Clin Respir J 2013; 7: 276-280

19 Schuetz P, Friedli N, Grolimund E, et al. Effect of hyperglycaemia on inflammatory and stress responses and clinical outcome of pneumonia in non-critical-care inpatients: results from an observational cohort study. Diabetologia 2014; 57: 275-284.

20 Van den Berghe G, Wouters P, Weekers F, et al. Intensive insulin therapy in critically ill patients. N Engl J Med 2001; 345: 1359-1367.

21 The NICE-SUGAR Study Investigators. Intensive versus conventional glucose control in critically ill patients. N Engl J Med 2009; 360: 1283-1297.

22 Di Yacovo S, Garcia-Vidal C, Viasus D, et al. Clinical features, etiology, and outcomes of community-acquired pneumonia in patients with diabetes mellitus. Medicine 2013; 92: 42-50.

23 Kornum JB, Thomsen RW, Riis A, et al. Diabetes, glycemic control, and risk of hospitalization with pneumonia. Diabetes Care 2008; 31: 1541-1545.

24 Yende S, van der Poll T, Lee M, et al. The influence of pre-existing diabetes mellitus on the host immune response and outcome of pneumonia: analysis of two multicenter cohort studies. Thorax 2010; 65: 870-877.

25 Falguera M, Pifarre R, Martin A, et al. Etiology and outcome of community-acquired pneumonia in patients with diabetes mellitus. Chest J 2005; 128: 3233-3239.

26 Diabetes Foreningen. Det National Diabetesregister [The National Diabetes Register]. www.diabetes.dk/presse/ diabetes-i-tal/det-nationale-diabetesregister.aspx

27 Langouche L, Vanhorebeek I, Vlasselaers D, et al. Intensive insulin therapy protects the endothelium of critically ill patients. J Clin Invest 2005; 115: 2277-2286.

28 Blum CA, Nigro N, Briel M, et al. Adjunct prednisone therapy for patients with community-acquired pneumonia: a multicentre, double-blind, randomised, placebo-controlled trial. Lancet 2015; 385: 1511-1518. 